INPLASY

PROTOCOL

To cite: Wang et al. Efficacy and Safety of Gegen Qinlian Decoction for Pediatric Diarrhea: A Systematic Review and Meta-analysis. Inplasy protocol 202180105. doi: 10.37766/inplasy2021.8.0105

Received: 26 August 2021

Published: 26 August 2021

Corresponding author: Yanjing Liu

liuyanjing666888@163.com

Author Affiliation:

Changchun University of

Traditional Chinese Medicine.

Support: 国家重点研发计划 (2017YFC1703206).

Review Stage at time of this submission: Completed but not published.

Conflicts of interest:

None declared.

\section{Efficacy and Safety of Gegen Qinlian Decoction for Pediatric Diarrhea: A Systematic Review and Meta-analysis}

Wang, D1; Bi, CR2; Li, YJ3; Jiang, HY4; Zhang, WP5; Liu, Y6; Liu, YJ7.

Review question / Objective: Objective. To evaluate the clinical efficacy and safety of Gegen Qinlian decoction in the treatment of pediatric diarrhea. Methods. A search for relevant RCTs was performed from which a systematic review and meta-analysis was conducted. Results. (1) Eleven trials involving 1126 patients were included in the meta-analysis. (2) Two trials recorded the adverse events. (3) The meta-analysis showed that compared with the control group, the experimental group has a significantly shorter duration of diarrhea in children [MD = -18.64, 95\% Cl (-23.76, -13.52), $\mathrm{P}<$ $0.00001]$, duration of fever [MD $=-19.43,95 \%$ Cl $(-25.76$, $-13.11), P<0.00001]$, duration of vomiting [MD $=-22.51,95 \%$ Cl (-29.92, -15.09), $P<0.00001]$, duration of correcting dehydration $[\mathrm{MD}=-23.35,95 \% \mathrm{Cl}(-35.48,-11.22), \mathrm{P}=0.0002]$, and the effective rate $[O R=4.64,95 \% \mathrm{Cl}(3.12,6.90), \mathrm{P}<0$. 00001]. Conclusion. There were significant differences in the clinical efficacy in the treatment of pediatric diarrhea between the experimental and control groups. Thus, Gegen Qinlian decoction may have certain advantages in the treatment of pediatric diarrhea.

INPLASY registration number: This protocol was registered with the International Platform of Registered Systematic Review and Meta-Analysis Protocols (INPLASY) on 26 August 2021 and was last updated on 26 August 2021 (registration number INPLASY202180105).

\section{INTRODUCTION}

Review question / Objective: Objective. To evaluate the clinical efficacy and safety of Gegen Qinlian decoction in the treatment of pediatric diarrhea. Methods. A search for relevant RCTs was performed from which a systematic review and metaanalysis was conducted. Results. (1) Eleven trials involving 1126 patients were included in the meta-analysis. (2) Two trials recorded the adverse events. (3) The meta-analysis 
showed that compared with the control group, the experimental group has a significantly shorter duration of diarrhea in children [MD $=-18.64,95 \%$ Cl $(-23.76$, $-13.52), P<0.00001]$, duration of fever [MD $=-19.43,95 \%$ Cl $(-25.76,-13.11), P<$ 0.00001 ], duration of vomiting [MD $=\mathbf{- 2 2 . 5 1}$, $95 \%$ Cl $(-29.92,-15.09), \quad P<0.00001]$, duration of correcting dehydration [MD = $-23.35,95 \%$ CI (-35.48, -11.22), $\mathrm{P}=0.0002]$, and the effective rate [OR=4.64, 95\% Cl (3.12, 6.90), $\mathrm{P}<0.00001]$. Conclusion. There were significant differences in the clinical efficacy in the treatment of pediatric diarrhea between the experimental and control groups. Thus, Gegen Qinlian decoction may have certain advantages in the treatment of pediatric diarrhea.

Condition being studied: Pediatric Diarrhea.

\section{METHODS}

Participant or population: The Review Manager 5.4 software was used to analyze the clinical data of 11 RCTs, involving 1126 participants. And all the trials are carried out in China.

Intervention: The control group was treated with conventional comprehensive treatment or combined with other treatments other than Gegen Qinlian Decoction. The treatment group was treated with Gegen Qinlian Decoction alone or Gegen Qinlian Decoction was added to the treatment plan of the control group.

Comparator: Duration of diarrhea; duration of fever; duration of vomiting; duration of correcting dehydration; the effective rate.

Study designs to be included: Inclusion Criteria (1) Objects: Literature published in domestic and international journals or conference papers related to GQD in the treatment of pediatric diarrhea.(2) Type: The article must be a RCT.(3) Treatment method: The control group was treated with conventional comprehensive treatment or combined with other treatments other than GQD. The treatment group was treated with GQD alone or GQD was added to the treatment plan of the control group.(4) Indicators (at least one): duration of diarrhea, fever, vomiting; time to correct dehydration; effective rate; adverse events.

Eligibility criteria: Exclusion Criteria(1) Does not meet the inclusion criteria.(2) RCT with incomplete data collection, inappropriate trial design, and inaccurate statistical methods.(3) Duplicate trials or data.(4) Experience summary, review, and case report;(5) Cell or animal experiments.

Information sources: Two researchers searched the literature in the China National Knowledge Infrastructure (CNKI; https://www.cnki.net), VIP database (http:// www.cquip.com), Wanfang database (https://www.wanfangdata.com.cn/ index.html), China Biomedical Database (CBM; http://www. sinomed.ac.cn), and Pubmed dat abase (https: / / pubmed.ncbi.nlm.nih.gov) according to the inclusion and exclusion criteria. The following terms were used to search the title, subject, and key words for relevant RCTs: "Gegen Qinlian decoction;" "children's diarrhea;" "diarrhea in children;" "pediatric diarrhea;" "infantile diarrhea;" "children's enteritis;" "enteritis in children;" and "pediatric enteritis." If necessary, the search included full text articles.

Main outcome(s): A total of 896 related articles were searched. By reading the title, abstract, and keywords, and according to the exclusion criteria, the articles that did not meet the standards were excluded. After re-screening, 11 RCTs were included. duration of diarrhea; duration of fever; duration of vomiting; duration of correcting dehydration; the effective rate; adverse events.

Quality assessment / Risk of bias analysis: The literature quality assessment of this study was conducted using the risk of bias table recommended by the Cochrane Collaboration. The table includes seven items: whether the randomized plan is clear; whether to hide the assignment; whether to blind during the test; whether to 
blind in the result analysis; whether the outcome data is complete; whether the results are selectively reported; and other sources of bias. The evaluation criteria are described as "yes" (low risk), "no" (high risk), or "unclear" (unclear). In the event of a disagreement during the evaluation process, the two researchers discuss the case first, and if the conflict cannot be resolved, the third researcher assists in the decision.

Strategy of data synthesis: Review Manager 5.4 software was used for statistical analysis. For continuous variables, the mean difference (MD) was used for statistical analysis, for dichotomous variables, the odds ratio (OR) was used, and the study confidence interval was set to $95 \%$. Heterogeneity analysis was performed using the 12 test. When $12 \leq 50 \%$ or $P \geq 0.05$ indicated no statistical heterogeneity, the fixed-effects model was used. Otherwise, a randomeffects model was used. The pediatric diarrhea curative effect of the treatment and control groups were compared using a forest diagram.

Subgroup analysis: Sensitivity (one-by-one excluding the RCT method) and subgroup analyses were used to clarify the source of heterogeneity.

Sensitivity analysis: Sensitivity (one-by-one excluding the RCT method) and subgroup analyses were used to clarify the source of heterogeneity.

Country(ies) involved: China.

Keywords: Gegen Qinlian Decoction; Pediatric Diarrhea; Systematic Review; Meta-analysis.

Contributions of each author:

Author 1 - Dan Wang.

Author 2 - Chaoran Bi.

Author 3 - Haiyan Jiang.

Author 4 - Yijing Li.

Author 5 - Wenping Zhang.

Author 6 - Yuan Liu.

Author 7 - Yanjing Liu. 